\section{PWE-243 REASSESSMENT OF CROHN'S DISEASE TREATED WITH 12 MONTHS OF ANTI-TNF THERAPY: A TERTIARY CENTRE EXPERIENCE}

doi:10.1136/gutjnl-2012-302514d.243

\begin{abstract}
${ }^{1,2} \mathrm{R}$ Dart, * ${ }^{3} \mathrm{~N}$ Griffin, ${ }^{1} \mathrm{~K}$ Taylor, ${ }^{1} \mathrm{~J}$ Duncan, ${ }^{1} \mathrm{M}$ Sastrillo, ${ }^{1} \mathrm{~S}$ Anderson, ${ }^{1,2} \mathrm{~J}$ Sanderson, 1,2P M Irving. 'Department of Gastroenterology, St Thomas' Hospital, London, UK; ${ }^{2}$ Diabetes and Nutritional Sciences Division, King's College London, London, UK; ${ }^{3}$ Department of Radiology, St Thomas' Hospital, London, UK
\end{abstract}

Introduction Anti-TNF therapy (ATT) is increasingly used in Crohn's disease (CD) in the UK. However, because of its expense, NICE guidance recommends that after a year of treatment, responders should be reassessed and withdrawal of treatment considered if they are in remission. We report our experience in a tertiary referral centre of reassessment after 1 year of ATT and of factors leading to continuation or withdrawal of treatment.

Methods We performed a 12-month retrospective review of patients with CD who had received ATT for $>12$ months by 31 December 2011. Reassessment was defined as having undergone one or more of the following investigations aimed at assessing disease activity: endoscopy, examination under anaesthesia, MRI or faecal calprotectin (FC). Results of investigations and outcome were recorded. Results 91 patients (infliximab $\mathrm{n}=55$, adalimumab $\mathrm{n}=36$ ) were included of whom $80 \%(73 / 91)$ had their disease reassessed. Five patients were withdrawn from treatment (of whom one has already relapsed) and five are pending trial of withdrawal; two patients met criteria for withdrawal but required continuation for extra-luminal disease such as arthropathy. 84 (92\%) continued therapy. 48 patients had endoscopic reassessment; mucosal healing $(\mathrm{MH})$ was demonstrated in $25 \%(n=12)$, non-ulcerating inflammation in $40 \%(n=19)$ and ulceration in $35 \%(n=17)$. Of 12 with $\mathrm{MH} 3$ withdrew from therapy and 3 are pending withdrawal. Of six patients who continue, two require ongoing ATT for arthropathy, three had radiologic evidence of activity, and one is undergoing further assessment. 24 patients had both endoscopy and MRI and 19 patients underwent MRI alone. Of the latter group, scans were normal in $21 \%(n=4)$, showed improvement but not resolution in $32 \%(n=6)$ and active disease in $47 \%(n=9)$. Of four normal scans, one patient was withdrawn, and 3 continue due to raised FC $(n=1)$ or raised CRP $(n=2)$. Disease was assessed by EUA in five patients, demonstrating active disease in four and quiescent disease in one who is pending trial of withdrawal. One patient continues on treatment on the basis of raised FC alone. One patient had mild mucosal inflammation on endoscopy and an unchanged MRI scan prior to withdrawal but relapsed within 4 months.

Conclusion Reassessment after at least 12 months of ATT showed ongoing disease activity in the vast majority (84\%). Withdrawal was considered appropriate in only $13 \%$. In patients with distal ileal and/ or colonic disease, endoscopy is currently our mainstay of reassessment while for those with small bowel disease interval change on MRI is used. The role of CRP and FC remain to be defined.

Competing interests None declared.

\section{PWE-244 DOES THROMBOPROPHYLAXIS IN ULCERATIVE COLITIS WORK?: DATA FROM THE UK IBD AUDIT}

doi:10.1136/gutjnl-2012-302514d.244

${ }^{1} \mathrm{R}$ W Lynch, ${ }^{*} \mathrm{M}$ Roughton, ${ }^{2} \mathrm{C}$ Down, I I Arnott. ${ }^{1} \mathrm{G} /$ Unit, Western General Hospital, Edinburgh, UK; ${ }^{2}$ CEEU, Royal College of Physicians, London, UK

Introduction Venous thromboembolism (VTE) has been long recognised as a complication of inflammatory bowel disease (IBD) with prevalence being reported at between 1.25 and $6.7 \%$ and a threefold increased risk being reported when compared to matched cohorts. It has led to recommendations that all patients admitted with acute flares of ulcerative colitis should be given prophylactic subcutaneous heparin although data of efficacy in an IBD population are lacking. We aim to assess the incidence, prophylaxis and possible aetiology of VTE in ulcerative colitis.

Methods We audited 3049 patients with UC. Median age was 42; there were 1421 females and 1628 males. 495 admissions were for elective surgery and there were 2504 emergency admissions of which 882 were considered to have severe disease. 202 Sites audited a median of 18 UC patients per site that were admitted with IBD between 1 September 2009 and 31 August 2010. Data collected contained specific questions on the administration of subcutaneous heparin and whether the patient had a thrombotic event during their admission. We also assessed whether type of admission, age, co-morbidities, disease severity, surgical operations, gender, steroid therapy and treatment response were related to VTE.

Results Of the 3049 patients admitted with ulcerative colitis, 2668 $(87.5 \%)$ patients were given prophylactic heparin. 66 (2.2\%) patients experienced thrombotic events during their admission. The rate of VTE among heparinised patients, 2.2\% (59/2668), did not differ significantly when compared to a rate of $1.8 \%$ (7/381) within the population who didn't receive prophylaxis $(p<0.8)$. VTE was associated with the need for surgery: $39.4 \%(26 / 66)$ of patients with VTE underwent surgery compared with surgery in $26.2 \%$ (781/ 2983) without VTE $(p<0.02)$. Of these 26 surgical patients within the VTE population, four of these patients' VTE were post-operative. When patients undergoing elective surgery are excluded VTE is associated with co-morbidity: $46.0 \%(23 / 50)$ of the VTE population compared to $32.4 \%(810 / 2504)$ in the non-VTE population $(p<0.03)$. There was no association of VTE with disease severity (severe disease in $42.0 \%(21 / 50)$ with DVT vs $34.4 \%(861 / 2504)$ without VTE, $\mathrm{p}<0.3$ ) or the use of steroids (steroids used in $96.0 \%$ (48/50) with VTE vs $89.6 \% \%(2244 / 2504)$ without VTE, $\mathrm{p}<0.08)$. Conclusion Although there was no difference in the frequency of VTE in patients given or not given heparin the numbers of VTE within this group are small. This analysis does however demonstrate that patients with co-morbidity and those undergoing surgery are at higher risk of VTE. Additional measures to prevent VTE should be considered in these patients such as a combination of heparin and compression stockings. All IBD patients admitted to hospital should continue to receive prophylactic subcutaneous heparin.

Competing interests None declared.

\section{PWE-245 ANTI-TNF $\alpha$ ANTIBODY THERAPY AND PARENTERAL CORTICOSTEROIDS DEMONSTRATE DISTINCT EFFECTS IN THE TREATMENT OF EXPERIMENTAL TRICHURIS MURIS-INDUCED CHRONIC COLITIS}

doi:10.1136/gutjnl-2012-302514d.245

${ }^{1} \mathrm{~S}$ E Levison, ${ }^{* 2} \mathrm{M} \mathrm{C}$ Little, ${ }^{2} \mathrm{R} \mathrm{K}$ Grencis, ${ }^{1} \mathrm{~J} \mathrm{~T}$ McLaughlin, ${ }^{1} \mathrm{~J} \mathrm{~L}$ Pennock. ${ }^{1}$ Translational Medicine; ${ }^{2}$ Manchester Immunology Group, University of Manchester, Manchester, UK

Introduction Anti-tumour necrosis factor (TNF) $\alpha$ antibody treatment and corticosteroid therapy represent central strategies in the management of Crohn's disease. Yet, over 30\% of patients fail to respond. Understanding the mechanism of effect for each therapy is complicated by disease heterogeneity, and the complexities of effector and regulatory immune cell responses. We characterised the biological and immunological effects of infliximab and hydrocortisone therapy in a genetically identical murine model of experimental colitis.

Methods Mice (AKR) susceptible to chronic Trichuris muris-induced colitis were infected with 300 T muris eggs. A single $5 \mathrm{mg} / \mathrm{kg}$ dose of Infliximab, or daily hydrocortisone treatment $(2 \mathrm{mg} / \mathrm{kg}$, ODS) were 